\title{
Post-Extract Effect (PEE)
}

\author{
Vijay Kothari ${ }^{1}$ \\ 1 Nirma University
}

Many multicomponent plant extracts/ polyherbal formulations can affect the pathogenic microorganisms in a variety of ways. Most such effects can not be detected through conventional antimicrobial assays (e.g. those for determination of MIC/ MBC). Effect of some potent extracts on microbial cells/ populations can last longer even after the extract-exposed cells are transferred subsequently into a fresh extract-free medium; and depending on the susceptiblity of the oraganims and potency of the extract, such antivirulence or growth-delaying effects may even last till next few subculturings in/on extract-free media. This phenomena can best be described as Post-Extract Effect (PEE), similar to Post-antibiotic Effect (PAE) described for some conventional antibiotics. However herbal or herbomineral formulations displaying multiplicity of targets are more likely to exert differnt types of PEEs on a variety of cellular/ physiological traits of the susceptible microbes, than single-molecule antibiotics with limited target spectrum. 